\title{
Stability of quantum states of finite macroscopic systems against classical noises, perturbations from environments, and local measurements
}

\author{
Akira Shimizu* and Takayuki Miyadera每 \\ Department of Basic Science, University of Tokyo, 3-8-1 Komaba, Tokyo 153-8902, Japan
}

\begin{abstract}
We study the stability of quantum states of macroscopic systems of finite volume $V$, against weak classical noises (WCNs), weak perturbations from environments (WPEs), and local measurements (LMs). We say that a pure state is 'fragile' if its decoherence rate is anomalously great, and 'stable against LMs' if the result of a LM is not affected by another LM at a distant point. By making full use of the locality and huge degrees of freedom, we show the following: (i) If square fluctuation of every additive operator is $O(V)$ or less for a pure state, then it is not fragile in any WCNs or WPEs. (ii) If square fluctuations of some additive operators are $O\left(V^{2}\right)$ for a pure state, then it is fragile in some WCNs or WPEs. (iii) If a state (pure or mixed) has the 'cluster property,' then it is stable against LMs, and vice versa. These results have many applications, among which we discuss the mechanism of symmetry breaking in finite systems.

PACS numbers: 03.65.Yz, 03.65.Ta, 11.30.Qc, 05.40.Ca
\end{abstract}

The stability of quantum states of macroscopic systems, which are subject to weak classical noises (WCNs) or weak perturbations from environments (WPEs), have been studied in many fields of physics as the decoherence problem [1]. However, most previous studies assumed that the principal system was describable by a small number of collective coordinates. Although such models might be applicable to some systems, applicability to general systems is questionable. As a result of the use of such models, the results depended strongly on the choices of the coordinates and the form of the interaction $\hat{H}_{\text {int }}$ between the principal system and a noise or an environment [1]. For example, a robust state for some $\hat{H}_{\text {int }}$ can become a fragile state for another $\hat{H}_{\text {int }}$. However, macroscopic physics and experiences strongly indicate that a more universal result should be drawn.

In this paper, we study the stability of quantum states of finite macroscopic systems against WCNs and WPEs. We also propose a new criterion of stability; the stability against local measurements (LMs). We study these stabilities using a general model with a macroscopic number of degrees of freedom $N$. In addition to the fact that $N$ is huge, we make full use of the locality [2,3] — 'additive' observables must be the sum of local observables over a macroscopic region, the interaction $\hat{H}_{\text {int }}$ must be local, and measurement must be local. By noticing these points, we derive general and universal results. Among many applications of the present theory, we discuss the mechanism of symmetry breaking in finite systems.

Macroscopic quantum systems: As usual, we are only interested in phenomena in some energy range $\Delta E$, and describe the system by an effective theory which correctly describes the system only in $\Delta E$. For a given $\Delta E$, let $\mathcal{M}$ be the number of many-body quantum states in that energy range. Then,

$$
N \sim \ln \mathcal{M}
$$

is the degrees of freedom of the effective theory. Note that $N$ can become a small number even for a system of many degrees of freedom when, e.g., a non-negligible energy gap exists in $\Delta E$, as in the cases of a heavy atom at a meV or lower energy range and SQUID systems at low temperatures. We here exclude such systems, because they are essentially systems of small degrees of freedom. Namely, we say that a system is macroscopic (for a given $\Delta E$ ) only when its $N$ is a macroscopic number. We further assume that the system extends homogeneously [4] over a volume $V$, and that boundary effects are negligible. Since $\Delta E$ sets a minimum length scale $\ell$,

$$
V \sim N \ell^{d}
$$

in $d$ dimension. We therefore say that $V$ is also macroscopic. We study the stability of states of such a macroscopic system, when it is subject to WCNs, WPEs, and LMs. The Hamiltonian $\hat{H}$ of the system can be a general one which has only short-range interactions.

Measures of the correlations between distant points: As we shall show later, correlations between distant points are important. As a measure of the correlations, we first consider the cluster property (CP). In infinite systems, a quantum state is said to have the $\mathrm{CP}$ if

$$
\langle\delta \hat{a}(x) \delta \hat{b}(y)\rangle \rightarrow 0 \text { as }|x-y| \rightarrow \infty
$$

for every local operators $\hat{a}(x)$ and $\hat{b}(y)$ at $x$ and $y$, respectively, where $\delta \hat{a}(x) \equiv \hat{a}(x)-\langle\hat{a}(x)\rangle$ and $\delta \hat{b}(y) \equiv$ $\hat{b}(y)-\langle\hat{b}(y)\rangle[5]$. Here, by a local operator at $x$ we mean a finite-order polynomial of field operators and their finiteorder derivatives at position $x$ [3]. We generalize the concept of the CP to the case of finite systems as follows [6]. For a small positive number $\epsilon$, we define a region $\Omega(\epsilon, x)$ by its complement $\Omega(\epsilon, x)^{c}$, which is the region of $y$ in which

$$
|\langle\delta \hat{a}(x) \delta \hat{b}(y)\rangle| \leq \epsilon \sqrt{\left\langle\delta \hat{a}^{\dagger}(x) \delta \hat{a}(x)\right\rangle\left\langle\delta \hat{b}^{\dagger}(y) \delta \hat{b}(y)\right\rangle}
$$

for every local operators $\hat{a}(x)$ and $\hat{b}(y)$. Let $\Omega(\epsilon) \equiv$ $\sup _{x}|\Omega(\epsilon, x)|$, where $|\Omega(\epsilon, x)|$ denotes the size of $\Omega(\epsilon, x)$. 
Intuitively, $\Omega(\epsilon)$ is the size of the region outside which correlations of every local operators becomes negligible. We consider a sequence of homogeneous [4] systems with various values of $V$ and associated states, where the shapes of $V$ 's are similar to each other. (For example, the ground states of many particles in spherical boxes with various sizes, with the same particle density.) We say that the states (for large $V$ ) of the sequence have the CP if $\Omega(\epsilon)$ for any $\epsilon>0$ becomes independent of $V$ for a sufficiently large $V$. This means that $\Omega(\epsilon) \ll V$ if one takes $V$ large enough. Note that a small number of Bell pairs do not destroy the $\mathrm{CP}$ : the lack of the $\mathrm{CP}$ means a macroscopic entanglement.

As a second measure, we consider fluctuations of additive quantities. A physical quantity $A$ is 'additive' if

$$
A=A^{(1)}+A^{(2)}
$$

when we regard the system as a composite system of subsystems 1 and 2 . Thermodynamics assumes that any states in a pure phase satisfies $\left\langle(\delta A)^{2}\right\rangle=o\left(V^{2}\right)$ for every additive quantity. In particular, if a state of (quantum or classical) system satisfies

$$
\left\langle(\delta A)^{2}\right\rangle \leq O(V)
$$

for every additive quantity, we call it a 'normallyfluctuating state' (NFS). In finite quantum systems, on the other hand, there exist pure states for which some of additive operators have anomalously-large fluctuations;

$$
\left\langle(\delta \hat{A})^{2}\right\rangle=O\left(V^{2}\right) .
$$

We call such a pure state an 'anomalously-fluctuating state' (AFS). The locality requires that additive operators of quantum systems must have the following form:

$$
\hat{A}=\sum_{x \in V} \hat{a}(x),
$$

where $\hat{a}(x)$ denotes a local operator at $x$. It is easy to show that an AFS does not have the CP, hence is entangled macroscopically. For infinite quantum systems, there is a well-known theorem: Any pure state has the $C P$ [2]. Therefore, AFSs converge (in the weak topology) into mixed states as $V \rightarrow \infty$, although they are pure states in finite systems [7]. Since AFSs are such unusual states, they are expected to be unstable in some sense. We now clarify in what sense, how, and why unstable.

Fragility: We say a quantum state is 'fragile' if its decoherence rate $\Gamma$ (see Eq. (3)) behaves as

$$
\Gamma \sim K V^{1+\delta},
$$

where $K$ is a function of microscopic parameters, and $\delta$ is a positive constant. To understand the meaning of the fragility, consider first the non-fragile case where $\delta=0$. In this case, $\Gamma / V$ is independent of $V$. This is a normal situation in the sense that the total decoherence rate $\Gamma$ is basically the sum of local decoherence rates, which are determined only by microscopic parameters. On the other hand, the case $\delta>0$ is an anomalous situation in which $\Gamma / V \sim K V^{\delta}$. Note that this can be very large even when $K$ is small, because, by definition, a macroscopic volume is huge. This means that a fragile quantum state decoheres due to a noise or environment at an anomalously great rate, even when the coupling constant between the system and the noise or environment is small.

Fragility under $W C N$ : The point of the present theory is the locality [2]. For the Hamiltonian $\hat{H}_{\text {int }}$ of the interaction with a classical noise, the locality requires that it should be the sum of local interactions [8];

$$
\hat{H}_{\mathrm{int}}=\lambda \sum_{x \in V} f(x, t) \hat{a}(x) .
$$

Here, $\lambda$ is a small positive constant, $f(x, t)$ is a random classical noise field with vanishing average $\overline{f(x, t)}=0$, and $\hat{a}(x)$ is a local operator at $x$. We assume that $\overline{f(x, t) f\left(x^{\prime}, t^{\prime}\right)}$ depends only on $x-x^{\prime}$ and $t-t^{\prime}$, and that its correlation time $\tau_{\mathrm{c}} \ll 1 / \Gamma[9]$. We denote the spectral intensity of $f$ by $g(k, \omega)[10]$, which is positive by definition. A pure state $|\Psi\rangle$ at $t=0$ evolves for $t>0$ by the total Hamiltonian $\hat{H}+\hat{H}_{\text {int }}$, and the density operator is given by

$$
\hat{\rho}(t) \equiv \overline{|\Psi(t)\rangle\langle\Psi(t)|} .
$$

Since we are interested in the dependence of $\Gamma$ on the initial state, we study an early time stage $\tau_{\mathrm{c}} \ll t \ll 1 / \Gamma$, and define $\Gamma$ as the increase rate of the $\alpha$-entropy of $\alpha=2$ in this time region;

$$
\Gamma \equiv-\left.\frac{1}{2} \frac{d}{d t} \ln \operatorname{Tr}\left[\hat{\rho}(t)^{2}\right]\right|_{\tau_{\mathrm{c}} \ll t \ll 1 / \Gamma} .
$$

Even when $\hat{H}_{\text {int }}=0,|\Psi\rangle$ generally evolves by $\hat{H}$. Since we are interested in the instability induced by $\hat{H}_{\text {int }}$, we consider states which do not evolve by $\hat{H}$ in this time region, i.e., $\exp (-i \hat{H} t)|\Psi\rangle \simeq \exp (-i\langle\hat{H}\rangle t)|\Psi\rangle$ for such t. (However, see [11].) Moreover, since we are interested in the case of weak noise, we evaluate $\Gamma$ to $O\left(\lambda^{2}\right)$. By dropping non-dissipative contributions from $\hat{H}_{\text {int }}$, because they can be absorbed in $\hat{H}$ as renormalization terms, we find

$$
\begin{aligned}
\Gamma & \simeq \lambda^{2} \sum_{k, n} g\left(k,\langle\hat{H}\rangle-\omega_{n}\right)\left|\left\langle n\left|\delta \hat{A}_{k}\right| \Psi\right\rangle\right|^{2} \\
& \equiv \lambda^{2} \sum_{k} g(k) \sum_{n}\left|\left\langle n\left|\delta \hat{A}_{k}\right| \Psi\right\rangle\right|^{2},
\end{aligned}
$$

where we have defined $g(k)$ by the last equality. Here, $|n\rangle$ is an eigenstate of $\hat{H}$, with eigenenergy $\omega_{n}$ (which may be degenerate), and $\delta \hat{A}_{k} \equiv \hat{A}_{k}-\left\langle\Psi\left|\hat{A}_{k}\right| \Psi\right\rangle$, where

$$
\hat{A}_{k} \equiv \sum_{x \in V} \hat{a}(x) e^{-i k x}
$$


Since $g\left(k,\langle\hat{H}\rangle-\omega_{n}\right)$ and $\left|\left\langle n\left|\delta \hat{A}_{k}\right| \Psi\right\rangle\right|^{2}$ are both positive, $g(k)$ may be interpreted as a typical (average) value of $g\left(k,\langle\hat{H}\rangle-\omega_{n}\right)$ for relevant $n$ 's. This interpretation would be good at least for the $V$ dependence, which is of our primary interest. We then obtain the simple formula;

$$
\Gamma \simeq \lambda^{2} \sum_{k} g(k)\left\langle\Psi\left|\delta \hat{A}_{k}^{\dagger} \delta \hat{A}_{k}\right| \Psi\right\rangle .
$$

Note that $\hat{A}_{k}$ is an additive operator because $\hat{a}(x) e^{-i k x}$ is a local operator. When $\hat{a}(x)$ is a spin operator, e.g., $\hat{A}_{k}$ for $k=\pi / \ell$ is the staggered magnetization.

When $|\Psi\rangle$ is an NFS, $\left\langle\Psi\left|\delta \hat{A}_{k}^{\dagger} \delta \hat{A}_{k}\right| \Psi\right\rangle \leq O(V)$ for any $\hat{A}_{k}$, hence

$$
\Gamma \lesssim \lambda^{2} O(V) \sum_{k} g(k)
$$

Since

$$
\sum_{k} g\left(k, \omega_{n}-\omega_{n^{\prime}}\right)=\int \overline{f(x, t) f(x, 0)} e^{i\left(\omega_{n}-\omega_{n^{\prime}}\right) t} d t
$$

does not depend on $V$, neither $\sum_{k} g(k)$ does. We thus find that NFSs are not fragile in any $W C N$. When $|\Psi\rangle$ is an AFS, on the other hand, $\left\langle\Psi\left|\delta \hat{A}_{k}^{\dagger} \delta \hat{A}_{k}\right| \Psi\right\rangle=O\left(V^{2}\right)$ for some $\hat{A}_{k}$, i.e., for some $\hat{a}(x)$ and some $k=k_{0}$. Hence, if $\hat{H}_{\text {int }}$ has a term that is composed of such $\hat{a}(x)$ 's, then

$$
\Gamma \simeq \lambda^{2} O\left(V^{2}\right) g\left(k_{0}\right)+\lambda^{2} O(V) \sum_{k \neq k_{0}} g(k),
$$

and the AFS becomes fragile if $g\left(k_{0}\right)=O\left(V^{-1+\delta}\right)$, where $\delta>0$. Therefore, an AFS is fragile in some $W C N$.

Fragility under WPE: The physical realities of noises are perturbations from environments. We obtain similar results for WPEs. From the locality, the interaction with an environment should be the sum of local interactions [8];

$$
\hat{H}_{\mathrm{int}}=\lambda \sum_{x \in V} \hat{f}(x) \hat{a}(x) .
$$

Here, $\hat{f}(x)$ and $\hat{a}(x)$ are local operators at $x$ of an environment and the principal system, respectively. For $\langle\hat{f}(x, t)\rangle_{\mathrm{E}}$ and $\left\langle\hat{f}(x, t) \hat{f}\left(x^{\prime}, t^{\prime}\right)\right\rangle_{\mathrm{E}}$ (in the interaction picture), where $\langle\cdots\rangle_{\mathrm{E}}$ denotes the expectation value for the state $\hat{\rho}_{\mathrm{E}}$ of the environment E, we assume the same properties as $\overline{f(x, t)}$ and $\overline{f(x, t) f\left(x^{\prime}, t^{\prime}\right)}$, respectively, of the WCN. The total Hamiltonian is

$$
\hat{H}+\hat{H}_{\mathrm{int}}+\hat{H}_{\mathrm{E}},
$$

where $\hat{H}_{\mathrm{E}}$ is the Hamiltonian of E. Taking the initial state $\hat{\rho}_{\text {total }}(0)$ as the product state $|\Psi\rangle\langle\Psi| \otimes \hat{\rho}_{\mathrm{E}}$, we evaluate the reduced density operator

$$
\hat{\rho}(t) \equiv \operatorname{Tr}_{\mathrm{E}}\left[\hat{\rho}_{\text {total }}(t)\right] .
$$

We then obtain the same result (雨), where $g(k, \omega)$ is now the spectral intensity derived from $\left\langle\hat{f}(x, t) \hat{f}\left(x^{\prime}, t^{\prime}\right)\right\rangle_{\mathrm{E}}$. Therefore, NFSs are not fragile under any WPE, while AFS are fragile under some WPE [12].

Summary of fragility: We have shown that NFSs are not fragile in any WCNs or WPEs. This should be contrasted with the results of most previous works, according to which a state could be either fragile or robust depending on the form of $\hat{H}_{\text {int }}$ [1]. Note that our results concern an approximate stability (i.e., non-fragility) against all possible WCNs or WPEs and $\hat{H}_{\text {int }}$ 's, whereas most previous works studied the exact stability against particular ones. We think that the former is more important in macroscopic systems because many types of WCNs or WPEs and $\hat{H}_{\text {int }}$ 's would coexist in real systems, and the exact stability against some of them could not exclude fragility to another. Regarding AFSs, on the other hand, our results show only that they are fragile in some WCN or WPE. In other words, for any AFS it is always possible to construct a noise (or an environment) and a weak local interaction with it in such a way that the AFS becomes fragile. These results do not guarantee the existence of the relevant noise (or an environment) and the relevant interaction in real physical systems. Since there is no theory that is general enough on WCNs or WPEs at present, we cannot draw a definite conclusion on whether AFSs are always fragile in real physical systems. It rather seems that, as we will discuss later, there may be some cases where some AFSs are non-fragile, in contradiction to naive expectations. This motivates us to explore the following new stability.

Stability against LMs: Suppose that one performs an ideal (von Neumann) measurement of a local observable $\hat{a}(x)$ at $t=t_{a}$ for a state $\hat{\rho}$ (pure or mixed) of a macroscopic system, and obtains a value $a$ with a finite probability $P(a) \neq 0$. Subsequently, one measures another local observable $\hat{b}(y)$ at a later time $t_{b}$ [13], and obtains a value $b$. Let $P(b ; a)$ be the probability that $b$ is obtained at $t_{b}$ under the condition that $a$ was obtained at $t_{a}$. On the other hand, one can measure $\hat{b}(y)$ at $t=t_{b}$ without performing the measurement of $\hat{a}(x)$ at $t_{a}$. Let $P(b)$ be the probability distribution of $b$ in this case. We say $\hat{\rho}$ is 'stable against local measurements' if for any $\varepsilon>0$

$$
|P(b ; a)-P(b)| \leq \varepsilon \text { for sufficiently large }|x-y|,
$$

for any local operators $\hat{a}(x)$ and $\hat{b}(y)$ and their eigenvalues $a$ and $b$ such that $P(a) \geq \varepsilon$. For the simplest case $t_{b} \rightarrow t_{a}$, we obtain the simple theorem: If $\hat{\rho}$ is stable against LMs then it has the CP, and that any state which has the $C P$ is stable against LMs. It follows, e.g., that any AFS is unstable against LMs.

To prove this theorem, we use the spectral decomposition 14;

$$
\hat{a}(x)=\sum_{a} a \hat{\mathcal{P}}_{a}(x),
$$


and similarly for $\hat{b}(y)$. Here, $\hat{\mathcal{P}}_{a}(x)$ denotes the projection operator corresponding to an eigenvalue $a$ of $\hat{a}(x)$. Note that $\left[\hat{\mathcal{P}}_{a}(x), \hat{\mathcal{P}}_{b}(y)\right]=0$ for $t_{b} \rightarrow t_{a}$. Since we are considering an effective theory in a finite energy range, we assume that ultraviolet divergences are absent: e.g., for any positive integer $m$,

$$
\left\langle\hat{a}(x)^{m}\right\rangle=\text { finite }
$$

for any local operator $\hat{a}(x)$. For $t_{b} \rightarrow t_{a}$, both $\mid P(b ; a)-$ $P(b) \mid \leq \varepsilon$ and $|P(a ; b)-P(a)| \leq \varepsilon$ are satisfied if $\hat{\rho}$ is stable against LMs. Expressing the probabilities by the projection operators, we obtain

$$
\begin{aligned}
& \left|\operatorname{Tr}\left[\hat{\rho} \hat{\mathcal{P}}_{a}(x) \hat{\mathcal{P}}_{b}(y)\right]-\operatorname{Tr}\left[\hat{\rho} \hat{\mathcal{P}}_{a}(x)\right] \operatorname{Tr}\left[\hat{\rho} \hat{\mathcal{P}}_{b}(y)\right]\right| \\
& \quad \leq \varepsilon \min (P(a), P(b))
\end{aligned}
$$

for $P(a), P(b) \geq \varepsilon$. Multiplying this equation by $|a b|$, and summing over $a$ and $b$ such that $P(a), P(b) \geq \varepsilon$, we obtain

$$
\left|\left\langle\delta \hat{a}_{\varepsilon}(x) \delta \hat{b}_{\varepsilon}(y)\right\rangle\right| \leq \varepsilon K .
$$

Here, $K$ is a finite positive number, which does not depend on $|x-y|$, and $\hat{a}_{\varepsilon}(x) \equiv \sum_{a}^{\prime} a \hat{\mathcal{P}}_{a}(x)$, where $\sum_{a}^{\prime}$ denotes the summation over $a$ such that $P(a) \geq \varepsilon$, and similarly for $\hat{b}_{\varepsilon}(y)$. By letting $\varepsilon \rightarrow 0$ (thus increasing $|x-y|$ accordingly), we obtain

$$
\left|\left\langle\delta \hat{a}_{\varepsilon}(x) \delta \hat{b}_{\varepsilon}(y)\right\rangle\right| \rightarrow|\langle\delta \hat{a}(x) \delta \hat{b}(y)\rangle| \rightarrow 0 .
$$

It is easy to show the CP from this. To prove the inverse, we take $\hat{a}(x)=\hat{\mathcal{P}}_{a}(x), \hat{b}(y)=\hat{\mathcal{P}}_{b}(y)$. Then, from (11),

$$
\begin{aligned}
\left|\left\langle\delta \hat{\mathcal{P}}_{a}(x) \delta \hat{\mathcal{P}}_{b}(y)\right\rangle\right| & \leq \epsilon \sqrt{P(a)(1-P(a)) P(b)(1-P(b))} \\
& \leq \epsilon \sqrt{P(a) P(b)}
\end{aligned}
$$

for sufficiently large $|x-y|$. Dividing this by $P(a)$ yields

$$
|P(b ; a)-P(b)| \leq \epsilon \sqrt{P(b) / P(a)} \leq \epsilon \sqrt{1 / P(a)} \leq \sqrt{\epsilon}
$$

for $P(a) \geq \epsilon$ (hence, also for $P(a) \geq \sqrt{\epsilon}$ ). We thus obtain the stability against LMs.

Applications: The above results have many applications, including quantum computers with many qubits [15], and non-equilibrium statistical physics. We here discuss the mechanism of symmetry breaking in finite systems, which has been a long-standing question for the following reasons. Consider a finite system that will exhibit a symmetry breaking if $V$ goes to infinity. Let $|\Psi\rangle_{V}$ be a state that approaches, as $V \rightarrow \infty$, a symmetry-breaking vacuum $|\Psi\rangle_{\infty}$ of the infinite system, in the sense that

$$
\lim _{V \rightarrow \infty}\left\langle\Psi_{V}|\hat{a}(x)| \Psi_{V}\right\rangle=\left\langle\Psi_{\infty}|\hat{a}(x)| \Psi_{\infty}\right\rangle
$$

for any local operator $\hat{a}(x)$. We call $|\Psi\rangle_{V}$ for large $V$ a pure-phase vacuum. It has a macroscopic value

$$
\left\langle\Psi_{V}|\hat{M}| \Psi_{V}\right\rangle=O(V)
$$

of an additive order parameter $\hat{M}$. In a mean-field approximation, pure-phase vacua have the lowest energy. However, it is always possible (see the example below) to construct a pure state(s) that does not break the symmetry, $\langle\hat{M}\rangle=0$, and has an equal or lower energy than pure-phase vacua [16,17]. Although such states cannot be pure in infinite systems, they can be pure in finite systems [2, 16, 17, 18]. When $[\hat{H}, \hat{M}] \neq 0$, in particular, the exact lowest-energy state is generally such a symmetric ground state [16,17. To lower the energy of a pure-phase vacuum, a symmetry-breaking field is necessary. However, an appropriate symmetry-breaking field would not always exist in real physical systems. For example, the symmetry-breaking field for antiferromagnets is a static staggered magnetic field, which alters its direction at the period exactly twice the lattice constant. It seems quite unlikely that such a field would always exist in laboratories.

Our results suggest the following new mechanisms of symmetry breaking in finite systems. From the wellknown theorem mentioned earlier, $|\Psi\rangle_{\infty}$ has the CP. Since $|\Psi\rangle_{V}$ approaches $|\Psi\rangle_{\infty}$, it also has the CP for large $V$. Hence, pure-phase vacua are not AFSs. On the other hand, $\left\langle\delta \hat{M}^{2}\right\rangle=O\left(V^{2}\right)$ for the symmetric ground state because it is composed primarily of a superposition of pure-phase vacua with different values of $\langle\hat{M}\rangle$ 16, 17. Namely, the symmetric ground state is an AFS, and thus is fragile in some WCN or WPE. Therefore, we expect that a pure-phase vacuum would be realized much more easily than the symmetric ground state. This mechanism may be called "environment-induced symmetry breaking," a special case of which was discussed for interacting many-bosons 18. For general systems, however, there is one delicate point: $g\left(k_{0}\right)$ of the relevant WCN or WPE might be $O(1 / V)$ in some of real systems [19. Then, Eq. (5) yields $\Gamma=O(V)$, and the symmetric ground state becomes non-fragile. In such a case, we must consider the stability against LMs: Even when the symmetric ground state is somehow realized at some time, it is changed into another state when one measures (or, 'looks' at) a relevant observable that is localized within only a tiny part of the system. Such drastic changes continue by repeating measurements of relevant observables, until the state becomes a pure-phase vacuum and the symmetry is broken. This mechanism may be called "measurement-induced symmetry breaking." We conjecture (and confirmed in several examples) that the number of LMs necessary for reducing an AFS to an NFS would be much less than $N$.

For example, if we regard the spins of the antiferromagnetic Ising model as quantum spins, the pure-phase vacua are the Néel states,

$$
\begin{aligned}
& \left|\Psi_{+}\right\rangle \equiv|\uparrow \downarrow \uparrow \cdots \downarrow\rangle, \\
& \left|\Psi_{-}\right\rangle \equiv|\downarrow \uparrow \downarrow \cdots \uparrow\rangle,
\end{aligned}
$$


for which the staggered magnetization

$$
\hat{M}_{\pi} \equiv \sum_{x} e^{i \pi x / \ell} \hat{\sigma}_{z}(x)
$$

is the order parameter;

$$
\left\langle\Psi_{ \pm}\left|\hat{M}_{\pi}\right| \Psi_{ \pm}\right\rangle= \pm V .
$$

On the other hand,

$$
|\Phi\rangle \equiv\left(\left|\Psi_{+}\right\rangle+\left|\Psi_{-}\right\rangle\right) / \sqrt{2}
$$

is an symmetric ground state. (In this simple model, $|\Phi\rangle$ is degenerate with $\left|\Psi_{ \pm}\right\rangle$. In more general models, the symmetric ground state is often the unique ground state [16, 17].) It is an AFS because $\left\langle\Phi\left|\hat{\delta} M_{\pi}^{2}\right| \Phi\right\rangle=V^{2}$. According to our results, $\left|\Psi_{ \pm}\right\rangle$are stable against LMs. For example, after measurement of $\hat{\sigma}_{x}$ of the first spin, $\left|\Psi_{+}\right\rangle$reduces to

$$
\frac{1}{\sqrt{2}}(|\uparrow\rangle+|\downarrow\rangle) \otimes|\downarrow \uparrow \cdots \downarrow\rangle \quad \text { when } \sigma_{x}=+1,
$$

or

$$
\frac{1}{\sqrt{2}}(|\uparrow\rangle-|\downarrow\rangle) \otimes|\downarrow \uparrow \cdots \downarrow\rangle \quad \text { when } \sigma_{x}=-1 .
$$

Hence, the result of subsequent measurement of any spin operator at a distant point is not affected at all by the first measurement. In contrast, $|\Phi\rangle$ are unstable against LMs, i.e., against measurement of some local spin operator. Namely, if the initial state is $|\Phi\rangle$, it is drastically altered by a measurement of only a tiny part of the system. For example, by measurement of $\hat{\sigma}_{z}$ of the first spin, $|\Phi\rangle$ reduces to either

$$
\left|\Psi_{+}\right\rangle \quad \text { when } \sigma_{z}=+1,
$$

or

$$
\left|\Psi_{-}\right\rangle \quad \text { when } \sigma_{z}=-1 .
$$

Hence, the results of subsequent measurements at distant points are drastically altered depending on the result of the first measurement. Note that the symmetric ground state turns into a pure-phase vacuum after the LM, and the symmetry is then broken. After that, the state alters only slightly by subsequent LMs, and the symmetry remains broken, because $\left|\Psi_{ \pm}\right\rangle$are stable against LMs. This may be the most general mechanism of symmetry breaking in finite systems.

In summary, we study the stabilities of quantum states of finite macroscopic systems, against weak classical noises, weak perturbations from environments, and local measurements. It is found that these stabilities are closely related to the cluster property (which describes the strength of spatial correlations of fluctuations of local observables) and fluctuations of additive operators (which are given by the sum of local operators over a macroscopic region). Note that the stabilities are defined as dynamical properties of an open system, whereas the cluster property and fluctuations of additive operators are defined as static properties of a closed system. Hence, it is non-trivial - may be surprising - that they are closely related to each other.

We thank I. Ojima, M. Ueda, H. Tasaki, and A. Ukena for discussions. This work is partially supported by Grant-in-Aid for Scientific Research.

* E-mail: shmz@ASone.c.u-tokyo.ac.jp

** Present address: Department of Information Sciences, Science University of Tokyo, Chiba 278-8510, Japan.

[1] See, e.g., papers in Quantum Coherence and Decoherence (eds. Y. A. Ono and K. Fujikawa, North-Holland, 1999).

[2] R. Haag, Local Quantum Physics (Springer, Berlin, 1992).

[3] To express and utilize the locality of the theory manifestly, we use a field theory throughout this paper.

[4] The homogeneity can be a generalized one: Our theory is applicable to, e.g., quantum computers [15], in which states for some $N$ are similar to states for another $N$ because they are generated by the same algorithm.

[5] The CP should not be confused with the absence of longrange order (LRO). In fact, symmetry-breaking vacua have both the LRO and CP [2,16,18. Moreover, domain walls, which destroy the LRO, do not destroy the CP.

[6] T. Miyadera, Thesis (University of Tokyo, 2001).

[7] In the Ising model discussed below, $\left|\Psi_{+}\right\rangle$and $\left|\Psi_{-}\right\rangle$become in the limit of $V \rightarrow \infty$ vectors of inequivalent Hilbert spaces $\mathcal{H}_{+}$and $\mathcal{H}_{-}$, respectively. Since there is no interference between them, $|\Phi\rangle \in \mathcal{H}_{+} \oplus \mathcal{H}_{-}$in this limit is equivalent to a classical mixture of $\left|\Psi_{+}\right\rangle$and $\left|\Psi_{-}\right\rangle$.

[8] Although we here describe the case where $f$ or $\hat{f}$ and $\hat{a}$ are real or hermitian, the generalization is straightforward.

[9] For a given $V$, this is satisfied for sufficiently small $\lambda$, e.g., $1 / \Gamma \sim 1 \mathrm{~ms}$ and $1 \mathrm{~ns}$, respectively, for a NFS and an AFS with $N=10^{6}$, whereas $\tau_{\mathrm{c}} \sim 1$ ps. Although $\Gamma$ varies with $\lambda$, we stress that $\Gamma$ for AFSs can be much larger than $\Gamma$ for NFSs by many orders of magnitude for any value of $\lambda$, as shown below.

[10] Since $f(x, t)$ for $x \notin V$ is irrelevant, the spatial Fourier transform is taken over $V$ to define $g(k, \omega)$, hence $k$ takes discrete values with separation $\sim V^{-1 / d}$.

[11] When this condition is violated around $t \sim t_{1}$ such that $\tau_{\mathrm{c}} \ll t_{1} \ll 1 / \Gamma$, we can apply formula (雨) again now to the evolved state $e^{-i \hat{H} t_{1}}|\Psi\rangle$, because the decoherence until $t_{1}$ does not affects $\Gamma$ after $t_{1}$ to $O\left(\lambda^{2}\right)$. We may repeat this for intervals $\left[t_{1}, t_{2}\right),\left[t_{2}, t_{3}\right), \cdots,\left[t_{M-1}, t_{M}\right)$ until some $t_{M} \ll$ $1 / \Gamma$. In cases of a rotating ferromagnet and a condensed state of bosons 17, the only quick evolution induced by $\hat{H}$ is the rotation of the direction and phase, respectively, of the order parameter. In such cases, $\Gamma$ in every interval is of the same order, and formula (4) gives the correct order of magnitude in the entire region of $\tau_{\mathrm{c}} \ll t \leq t_{M}$, although $e^{-i \hat{H} t}|\Psi\rangle \simeq e^{-i\langle\hat{H}\rangle t}|\Psi\rangle$ is not satisfied for $t>t_{1}$. 
[12] A special case of this result was obtained by G. M. Palma, K.-A. Suominen and A. K. Ekert, Proc. Roy. Soc. Lond. A 452, 567 (1996).

[13] $\left(x, t_{a}\right)$ and $\left(y, t_{b}\right)$ are separated either time- or space-like.

[14] The case of continuous eigenvalues can be discussed in a similar manner.

[15] A. Shimizu et al., unpublished.

[16] P. Horsh and W. von der Linden, Z. Phys. B72, 181 (1988); T. Koma and H. Tasaki, J. Stat. Phys. 76, 745 (1994).

[17] A. Shimizu and T. Miyadera, Phys. Rev. E64, 056121 (2001).

[18] A. Shimizu and T. Miyadera, Phys. Rev. Lett. 85, 688 (2000); A. Shimizu and T. Miyadera, J. Phys. Soc. Jpn. 71, 56 (2002).

[19] For example, electromagnetic noises at $4 \mathrm{~K}$ contribute only to $k=0$ component of $g(k)$ if the diameter of the system is less than $1 \mathrm{~cm}[10]$. Since $\sum_{k} g(k)=O\left(V^{0}\right), g(0)=O\left(V^{0}\right)$ whereas $g(k)=O(1 / V)$ for $k \gg 1 \mathrm{~cm}^{-1}$. 